\title{
Review
}

Ming Guo*

\section{Research progress of follicular cytotoxic T cells in HIV infection}

DOI: 10.2478/ii-2018-0006

Received January 28, 2018; accepted February 06, 2018; published online April 10, 2018

\begin{abstract}
Recently, a new type of CD8 ${ }^{+}$T-cell subset, namely, the chemokine (C-X-C motif) receptor 5 (CXCR5 ${ }^{+}$) cluster of differentiation (CD8+) T-cell subset (also called the follicular cytotoxic T-cell (TFC) subgroup), has been discovered around B-cell follicles. The discovery has aroused widespread interest. However, the processes and mechanisms of TFCs taking part in the immune response of the germinal center and their specific roles must still be clearly identified. This article reviews domestic and foreign studies on factors regulating the phenotype, physiological functions, maturity, and differentiation of TFCs and roles and clinical significance of these cells in HIV infection. This review has shown good application prospects for TFCs. The author believes that further studies on TFCs can provide another tool for cytotherapy to control or cure chronic viral infections or tumors.
\end{abstract}

Keywords: follicular cytotoxic T cells, HIV infection, progress

During viral infections, antigen-specific $\mathrm{CD} 8^{+} \mathrm{T}$ cells, which are mainly present in secondary lymphoid organs, rapidly differentiate into effector T cells, which eliminate infections. On the other hand, $\mathrm{CD}^{+} \mathrm{T}$ cells differentiate into follicular helper T cells (TFHs), which enhance the ability of B cells to produce protective antibodies. In some cases, viruses evade immune control and clearance, resulting in persistent viral infection and replication. For instance, HIV can evade specific immune cells, making TFHs important targets for persistent HIV infection and thus becoming a major obstacle to finding a functional HIV cure [1]. As TFHs mainly exist in B-cell follicles, intensive studies on the location, phenotypes, and functions of cytotoxic effector T cells in B-cell follicles bear significance in developing new measures for specific clearance of HIV.

Recent studies have shown the existence of a new $\mathrm{CD}^{+} \mathrm{T}$-cell subset, called the CXCR5 ${ }^{+} \mathrm{CD}^{+} \mathrm{T}$ cell subset, also known as the follicular cytotoxic T cell (TFC) subgroup, around B-cell follicles; this subset has attracted significant attention worldwide. CXCR5 is expressed in all B cells, TFHs, and TFCs [2,3]. At present, the only known ligand for CXCR5 is chemokine (C-X-C motif) ligand $_{13}$ (CXCL13), which is primarily produced by stromal cells and follicular dendritic cells surrounding B-cell follicles [4]. TFHs that are present in B-cell follicles exert an effective humoral immune response through production of secondary antibodies $[5,6]$. However, studies have neither identified the immune response process or mechanism of the germinal center nor the roles of TFCs. This article reviews current studies on TFCs and their roles in HIV infection.

\section{Phenotypes of TFCs}

CXCR5 is specifically expressed in and required by TFCs to move around B-cell follicles. Chemokine receptor 5 (CCR5) is, but not chemokine CCR7, also expressed in TFCs. TFHs that home to lymphoid tissues via CCR7 suggest their different homing or migration mechanisms compared with those of TFCs.

The $5^{\prime}$ region of CXCR5 gene contains three regulatory elements, and CXCR5 expression is closely related to these regulatory elements. B lymphocyte-induced maturation protein (Blimp1) and the transcription factor

Department of Hematology, Second Affiliated Hospital, Beijing University of Traditional Chinese Medicine,

Beijing 100078, China

*Correspondence: Ming Guo, E-mail: redsnow_g@126.com 
E2A regulate the transcription of CXCR5 after combining with these regulatory elements [7]. E2A activates the transcription of CXCR5, whereas Blimp1 causes its inhibition. In addition to directly regulating the expression of CXCR5, transcription factors Blimp1 and E2A may also form transcription pathways with inhibitor of differentiation 2 (Id2) and Id3 profiling of B cell lymphoma-6 (Bc16), T-cell factor 1 (TCF-1), and E2A, thereby controlling expression of CXCR5 and formation of TFCs. Expressions of inhibitory molecules such as T cell immunoglobulin domain and mucin domain protein 3 (Tim3), CD39, and 2B4 are downregulated in TFCs. However, downregulated molecules, relative to those in cytotoxic T cells (CTLs), show no difference between $\mathrm{CXCR}^{+} \mathrm{CD}^{+} \mathrm{T}$ cells and CTLs in expression of programmed cell death protein 1 (PD-1) [8].

Another feature of TFCs is high expression of memory-related molecules, such as CD62L, interleukin (IL)-7R, and TCF-1, and low expression of perforin, which may be partially related to the low expression of Blimp1. Blimp1 is associated with expressions of various cytotoxic molecules [9], and as an inhibitor of the differentiation of memory T cells, it also downregulates Sell (encoding CD62L), IL-7R, and TCF [10]. Therefore, upregulation of Bcl6 and downregulation of Blimp1 in differentiation of TFCs lead to elevated expression of CXCR5, increase the likelihood of TFCs entering into the follicular regions of B cells, and reduce cytotoxic effector functions of cells. TFCs and CTL subset differ in expressions of Toll-like receptors (TLRs). TLRs are molecules that are closely related to innate immune reactions, but their roles in CD8+ T cells have not been identified [11].

Some similarities and differences can be observed in phenotypes of TFCs in peripheral blood and lymphoid tissues. In TFCs of lymphoid tissues, expressions of co-stimulatory molecules, CD27 and CD28, are upregulated. The activated molecules CD69 and CD95 and memory molecule CD45RO are expressed, whereas CD7 is downregulated. This condition indicates that these cells are antigen-primed T cells. IL-7 receptor CD127 is expressed in TFCs on a fraction of lymphoid tissues. TFCs in peripheral blood show expression of CD127 and absence of CD69. This phenomenon indicates reduced activation of circulating CXCR5 ${ }^{+} \mathrm{CD} 8^{+} \mathrm{T}$ cells. CD62L is hardly expressed in TFCs in lymphoid tissues probably due to downregulation of adhesion molecules while entering the lymphoid tissues. CD62L is upregulated in $\mathrm{CXCR}^{+} \mathrm{CD}^{+} \mathrm{T}$ cells of peripheral blood. This condition indicates that CD62L can adhere to endothelial venules. CCR5 is expressed in TFCs in approximately $50 \%$ of peripheral blood, but the level is lower than that in lymph nodes.

Several unknown factors influence the relationship between detailed phenotypic characteristics and functions of TFCs. This relationship will become one of the key research thrusts for TFCs in the future.

\section{Physiological functions of TFCs}

TFCs with stem cell-like features slowly renew themselves and produce terminally differentiated effector $\mathrm{CD}^{+} \mathrm{T}$ cells, which may exist in lymphoid and non-lymphoid tissues. TFCs in peripheral blood and lymphoid tissues may also produce interferon (IFN)- $\gamma$, tumor necrosis factor (TNF)- $\alpha$, IL-2, and granzyme A, instead of the cytotoxic protein perforin [12]. This phenomenon suggests that TFCs possess the features of non-cytolytic effector memory cells. Compared with CXCR5 $\mathrm{CD}^{+} \mathrm{T}$ cells, the TFC subgroup possesses more capacity to produce IFN- $\gamma$ and TNF- $\alpha$. The degranulation marker CD107 is expressed in about $30 \%$ of TFCs, whereas CD107 staining of CXCR5 CD8 ${ }^{+} \mathrm{T}$ cells is almost constantly negative, indicating that TFCs feature high antiviral activity. High-level effector molecules, such as perforin, are secreted from CXCR5 ${ }^{-} \mathrm{CD} 8^{+} \mathrm{T}$ cells but are expressed with very weak proliferation without IL-2 or TNF. Such condition suggests that CXCR5 $\mathrm{CD}^{+} \mathrm{T}$ cells are terminally differentiated.

TFCs play a supporting role in the survival and functions of follicular B cells. However, TFHs do not strongly support follicular B cells. CD70, OX40, and inducible T-cell costimulator (ICOS), which are produced by TFC activation, play important roles through their interaction with B cells. TFCs perform supplementary roles in immunoglobulin $\mathrm{G}$ production in follicular B cells. The effective interaction between TFCs and B cells requires molecule pairs, such as $\mathrm{CD} 27, \mathrm{CD} 70$, and OX40, in addition to the interaction of homologous T-cell receptor and major histocompatibility complex [13]. Similarly, B cells are highly important in the survival and functions of TFCs. Studies discovered that when comparing TFCs transfected into infected wild-type mice 
and B-cell-deficient mice, poor survival was observed in TFCs in B-cell-deficient mice [12]. Both TFC toxicity and production ability of cytokines were reduced in B-cell-deficient mice. High-level chemokines such as X-C motif chemokine ligand 1 (Xcl1) are also expressed in TFCs [8], improving the interactions with dendritic cells in lymph nodes.

As described earlier, the interactions among TFCs, TFHs, and B cells play crucial roles in normal functioning of such cells. However, the specific mechanism of interactions among these groups of cells remains poorly understood and is considered a research priority in the future.

\section{Factors regulating maturity and differentiation of TFCs}

The Id2/E2A axis plays an important role in regulating the functions and differentiation of TFCs. He et al. [12] observed that by comparing the transcriptomes of $\mathrm{CXCR}^{+} \mathrm{CD}^{+} \mathrm{T}$ cells and $\mathrm{CXCR} 5^{-} \mathrm{CD} 8^{+} \mathrm{T}$ cells, proteins of the TNF family, their receptors, and specific chemokine receptors are highly expressed in $\mathrm{CXCR}^{+} \mathrm{CD}^{+} \mathrm{T}$ cells. The same researchers also discovered that E2A is significantly enriched in $\mathrm{CXCR}^{+} \mathrm{CD}^{+} \mathrm{T}$ cells, and that low-level Id 2 in $\mathrm{CXCR}^{+} \mathrm{CD}^{+} \mathrm{T}$ cells inhibits transcriptional activity of E2A. In chronic infections, the number and frequency of $\mathrm{CXCR5}^{+} \mathrm{CD}^{+} \mathrm{T}$ cells in mice with Id2 $2^{-/-}$significantly increase, whereas $\mathrm{CXCR} 5^{+} \mathrm{CD} 8^{+} \mathrm{T}$ cells exhibit stronger effector functions after removal of Id2. CXCR5 in CD8 ${ }^{+} \mathrm{T}$ cells is significantly upregulated with overexpression E2A, and when Id2 is also overexpressed, the effect will be reduced. Notably, cells with overexpressed E2A contain downregulated PD-1 and elevated secretion of CD107 and cytokines. These results showed that the Id2/E2A axis regulates differentiation and functions of $\mathrm{CXCR}^{+} \mathrm{CD}^{+} \mathrm{T}$ cells. In addition to the regulation stated earlier, production of $\mathrm{CXCR}^{+} \mathrm{CD}^{+} \mathrm{T}$ cells is highly dependent on the thymus.

In addition to the major regulatory functions of the E2A/Id2 regulatory axis, the transcription factors Blimp1, E2A, Bcl6, and TCF-1 and E2A profilins Id2 and Id3 jointly form transcription pathways and control the formation of TFCs. E2A activates transcription of CXCR5, whereas Blimp1 inhibits the process. In TFCs, removal of Id3 leads to upregulation of CXCR5. On the other hand, overexpression of Id3 inhibits expression of CXCR5. As both Blimp1 and E2A regulate CXCR5 expression in TFHs and B cells, the pattern is possibly a universal one. This pattern also determines CXCR5 expression and function of such cells.

Factors that further influence maturity and differentiation of TFCs may provide additional insights into TFCs. Such variables affect production and functions of TFCs by influencing regulation of key genes or molecules in TFCs. The detailed regulation process and pathway molecules are some of the most important research directions for TFCs in the future.

\section{Roles and clinical significance of TFCs in HIV infection}

TFCs refer to a major subset of cells that exert controlling effects on chronic viral infection with extensive depletion of CTLs [7]. In HIV infection, CTLs act as major anti-HIV effector cells, playing an important role in killing HIV-infected cells and preventing intercellular spread of HIV. As the disease progresses, HIV-specific CTLs are manifested with progressive failure. Some scientific research groups, both local and abroad, have conducted preliminary discussions regarding the roles of TFCs in chronic HIV infection.

He et al. [12] initially studied the relationship between TFCs in untreated HIV-infected patients and disease progression; they discovered that in the plasma of untreated HIV-infected patients, viral load is inversely proportional to the number of TFCs. HIV-specific TFCs can be detected in lymph nodes, and their expressions are higher than those of IFN- $\gamma$, TNF- $\alpha$, and CD107, which are secreted from CXCR5 ${ }^{-}$CD $8^{+}$T cells. In HIV infection, expression of Id 2 in $\mathrm{CXCR}^{+} \mathrm{CD}^{+} \mathrm{T}$ cells is lower than that in CXCR5 $\mathrm{CD}^{+} \mathrm{T}$ cells. In untreated HIV-infected patients, TFCs localize around HIV-infected TFHs in follicles. These findings indicate that these cells play an important role in controlling HIV infection around follicles. In chronic simian immunodeficiency virus or HIV infection, TFHs at follicular sites of B cells act as important HIV-infected and toxigenic 
cells. However, extremely few TFCs exist at these sites, thus resulting in persistent infection around B-cell follicles. The adoptive transfer of TFCs or intervention of the E2A/Id2 axis may effectively clear HIV infection in B-cell follicles [12].

In chronic lymphocytic choriomeningitis virus infection and tumors, immune response of $\mathrm{T}$ cells may be improved by blocking the PD-1 inhibitory pathway [1,14-15]. CXCR5 ${ }^{+}$and $\mathrm{CXCR}^{-} \mathrm{CD}^{+} \mathrm{T}$ cells were transcribed in mice with chronic viral infection, after which PD-1 blocker was applied. Results showed relatively weak effector functions of PD-1 blocker on $\mathrm{CXCR}^{-} \mathrm{CD}^{+} \mathrm{T}$ cells. However, $\mathrm{CXCR} 5^{+} \mathrm{CD}^{+} \mathrm{T}$ cells responded more actively to PD-1 blocker. PD-1 blocker may significantly increase differentiation of CXCR5 ${ }^{+} \mathrm{CD}^{+} \mathrm{T}$ cells into $\mathrm{CXCR}^{-} \mathrm{CD}^{+} \mathrm{T}$ cells and effector functions of such cells by more than 30 -folds [8]. These findings suggest that PD-1 blocker may boost proliferation and effector functions of $\mathrm{CXCR} 5^{+} \mathrm{CD} 8^{+} \mathrm{T}$ cells. Whether proliferation and effector functions of $\mathrm{CXCR} 5^{+} \mathrm{CD} 8^{+} \mathrm{T}$ cells can be enhanced by adding PD-1 blocker in HIV infection should be further explored.

In terms of persistent viral infection, TFCs are commonly assumed as important factors for killing HIV viruses. Studies have shown that TFHs are one of the most important targets for HIV latency and that TFCs are insufficient for causing HIV infection or increasing the number of latent viruses in TFHs [1,16-17]. Thus, thoroughly understanding the roles of TFCs in HIV infection and probing further into the roles of TFCs in clearing HIV viruses and latent HIV viruses from TFHs may bear significance in the design of new annihilation procedures for HIV repositories.

In summary, TFCs refer to a newly discovered class of $C D 8^{+} \mathrm{T}$ cell subset, which is mainly present around follicular B cells. With the depletion of specific CTLs in chronic infections and tumors, TFCs become the most active cell subset in killing viruses. Phenotypes, functions, sources, production mechanisms, or reactions of TFCs with other cells remain incompletely understood. Intensive studies should be conducted to better understand TFCs, which have shown good application prospects. Further studies on such cells may result in a novel method for controlling or curing chronic viral infections or tumors.

Acknowledgments: None.

Conflict of interest: The author states no conflicts of interest.

\section{References}

[1] Banga R., Procopio F.A., Noto A., Pollakis G., Cavassini M., Ohmiti K., et al., PD-1+ and follicular helper T cells are responsible for persistent HIV-1 transcription in treated aviremic individuals, Nat. Med., 2016, 22(7), 754-761.

[2] Müller G., Höpken U.E., Lipp M., The impact of CCR7 and CXCR5 on lymphoid organ development and systemic immunity, Immunol. Rev., 2003, 195(1), 117-135.

[3] Quigley M.F., Gonzalez V.D., Granath A., Andersson J., Sandberg J.K., CXCR5+, Eur. J. Immunol., 2007, 37(12), $3352-3362$.

[4] Ansel K.M., Ngo V.N., Hyman P.L., Luther S.A., Förster R., Sedgwick J.D., et al., A chemokine-driven positive feedback loop organizes lymphoid follicles, Nature, 2000, 406(6793), 309-314.

[5] Breitfeld D., Ohl L., Kremmer E., Ellwart J., Sallusto F., Lipp M., et al., Follicular B helper T cells express CXC chemokine receptor 5, localize to B cell follicles, and support immunoglobulin production, J. Exp. Med., 2000, 192(11), 1545-1552.

[6] Schaerli P., Willimann K., Lang A.B., Lipp M., Loetscher P., Moser B., CXC chemokine receptor 5 expression defines follicular homing T cells with B cell helper function, J. Exp. Med., 2000, 192(11), 1553-1562.

[7] Leong Y.A., Chen Y., Ong H.S., Wu D., Man K., Deleage C., et al., CXCR5(+) follicular cytotoxic T cells control viral infection in B cell follicles, Nat. Immunol., 2016, 17(10), 1187-1196.

[8] Im S.J., Hashimoto M., Gerner M.Y., Lee J., Kissick H.T., Burger M.C., et al., Defining CD8+ T cells that provide the proliferative burst after PD-1 therapy, Nature, 2016, 537(7620), 417-421.

[9] He J., Tsai L.M., Leong Y.A., Hu X., Ma C.S., Chevalier N., et al., Circulating precursor CCR7(lo) PD-1(hi) CXCR5+ CD4+ T cells indicate Tfh cell activity and promote antibody responses upon antigen reexposure, Immunity, 2013, 39(4), 770-781.

[10] Nance J.P., Bélanger S., Johnston R.J., Takemori T., Crotty S., Cutting edge: T follicular helper cell differentiation is defective in the absence of Bcl6 BTB repressor domain function, J. Immunol., 2015, 194(12), 5599-5603.

[11] Takeda K., Kaisho T., Akira S., Toll-like receptors, Annu. Rev. Immunol., 2003, 21(21), 335-376. 
[12] He R., Hou S., Liu C., Zhang A., Bai Q., Han M., et al., Follicular CXCR5-expressing CD8+ T cells curtail chronic viral infection, Nature, 2016, 537(7620), 412-428.

[13] Croft M., Co-stimulatory members of the TNFR family: keys to effective T-cell immunity, Nat. Rev. Immunol., 2003, 3(8), 609-620.

[14] Sharma P., Allison J.P., The future of immune checkpoint therapy, Science, 2015, 348(6230), 56-61.

[15] Barber D.L., Wherry E.J., Masopust D., Zhu B., Allison J.P., Sharpe A.H., et al., Restoring function in exhausted CD8 T cells during chronic viral infection, Nature, 2006, 439(7077), 682-687.

[16] Hong J.J., Amancha P.K., Rogers K., Ansari A.A., Villinger F., Spatial alterations between CD4+ T follicular helper, B, and CD8+ T cells during simian immunodeficiency virus infection: T/B cell homeostasis, activation, and potential mechanism for viral escape, J. Immunol., 2012, 188(7), 3247-3256.

[17] Fukazawa Y., Lum R., Okoye A.A., Park H., Matsuda K., Bae J.Y., et al., B cell follicle sanctuary permits persistent productive simian immunodeficiency virus infection in elite controllers, Nat. Med., 2015, 21(2), 132-139. 